\title{
Effect of rosuvastatin on atrial structural remodeling in rabbits with myocardial infarction
}

\author{
WEIJIE WANG ${ }^{1,2^{*}}$, YUJIAO ZHANG ${ }^{1,3^{*}}$, MEI GAO $^{1}$, JIANGRONG WANG $^{1}$ and YINGLONG HOU $^{1}$ \\ ${ }^{1}$ Department of Cardiology, Shandong Provincial Qianfoshan Hospital, Shandong University, Jinan, \\ Shandong 250014; ${ }^{2}$ Department of ICU, People's Hospital of Rizhao, Rizhao, Shandong 276826; \\ ${ }^{3}$ School of Medicine, Shandong University, Jinan, Shandong 250012, P.R. China
}

Received August 20, 2014; Accepted September 25, 2014

DOI: $10.3892 /$ br.2014.366

\begin{abstract}
The present study aimed to investigate the effect of rosuvastatin on atrial structural remodeling in rabbits with myocardial infarction (MI). The rabbits were randomly divided into 4 groups: The sham operation group ( $\mathrm{S} ; \mathrm{n}=8)$, the MI group (MI; $n=7)$, the group with low-dose rosuvastatin $\left[R_{s}\right.$; $2.5 \mathrm{mg} /(\mathrm{kg} / \mathrm{d}), \mathrm{n}=7]$ and the group with high-dose rosuvastatin $\left[\mathrm{R}_{1}, 5 \mathrm{mg} /(\mathrm{kg} / \mathrm{d}), \mathrm{n}=8\right]$. A rabbit MI model was established via ligation of the anterior descending coronary artery. After 8 weeks of intervention, the left atrial diameter (LAD), atrial collagen content, expression level of type I collagen and left ventricular ejection fraction (LVEF) were measured. LAD in the MI group was significantly larger than the $S, R_{s}$ and $\mathrm{R}_{1}$ groups after 8 weeks. The left atrial collagen content of the MI group was also significantly greater than those observed in the other 3 groups. The expression of type I collagen in the MI group was significantly increased compared to the other 3 groups. However, LVEF of the MI group was lower compared to the other 3 groups. There were no significant differences between the $R_{s}$ and $R_{1}$ groups in the above indexes. In conclusion, rosuvastatin can improve post-MI atrial structural remodeling.
\end{abstract}

\section{Introduction}

In addition to their role in controlling lipid levels, the pleiotropic effects of statins, including the anti-arrhythmia effects and prevention of cardiac remodeling, have attracted significant attention $(1,2)$. A large number of studies have focused on left ventricular remodeling following myocardial infarction

Correspondence to: Dr Yinglong Hou, Department of Cardiology, Shandong Provincial Qianfoshan Hospital, Shandong University, 16766 Jingshi Road, Jinan, Shandong 250014, P.R. China

E-mail: houyinglong2013@hotmail.com

*Contributed equally

Key words: rosuvastatin, myocardial infarction, atrial structural remodeling, rabbit
(MI) and the treatment effect of statins (3-6). In addition to ventricular arrhythmia, atrial arrhythmia and in particular, atrial fibrillation (AF), represent a common occurrence following MI (7) and studies have shown that statins can exert preventive and therapeutic effects on atrial arrhythmias (8). With the goal to reveal the mechanisms underlying the preventive and therapeutic effects of statins on post-MI atrial arrhythmia, the changes were investigated in post-MI atrial structural remodeling mediated by rosuvastatin in the present study.

\section{Materials and methods}

Drugs and reagents. The main drugs and reagents used in the study included rosuvastatin (H20060406; AstraZeneca, London, UK), the hydroxyproline assay kit and Masson staining kit (Njjcbio, Nanjing, China), an anti-type I collagen antibody (Abcam, Cambridge, UK) and the Bradford Protein Assay kit (Blkwbio, Beijing, China).

Animals and grouping. Healthy New Zealand white rabbits of either gender, weighing $2.2 \pm 0.3 \mathrm{~kg}$, were purchased from the Experimental Animal Center of Shandong University of Traditional Chinese Medicine (Shandong, China). The animal research protocol complied with 'The Guide for the Care of Use of Laboratory Animals' published by the National Institutes of Health (publication no. 85-23, revised 1996) and was approved by the Animal Care Committee of Medical College of Shandong University. The 30 surviving rabbits were randomly divided into the sham operation control group (S; $\mathrm{n}=8$ ), the MI group (MI; $\mathrm{n}=7$ ), the low-dose rosuvastatin group $\left[\mathrm{R}_{\mathrm{s}} ; 2.5 \mathrm{mg} /(\mathrm{kg} / \mathrm{d}), \mathrm{n}=7\right]$ and the high-dose rosuvastatin group $\left[\mathrm{R}_{1} ; 5 \mathrm{mg} /(\mathrm{kg} / \mathrm{d}), \mathrm{n}=8\right]$. The $\mathrm{S}$ group received thoracotomy only. Ligation of the left anterior descending coronary artery was performed to induce MI. The rabbits in the $R_{s}$ and $R_{1}$ groups received intragastric administration of rosuvastatin following MI for 8 weeks, while the rabbits in the S and MI groups did not receive any drug intervention.

Establishment of the rabbit MI model. The rabbits were anesthetized with $3 \%$ sodium pentobarbital $(40 \mathrm{mg} / \mathrm{kg})$ by intraperitoneal injection and were fixed on a small animal surgery table. Following a routine preoperative electrocardiogram 
(ECG), a longitudinal incision was made along the left sternal border, using the 3rd and 4th intercostal spaces as a midpoint, to fully expose the heart. Subsequent to opening the pericardium, the heart was lifted to expose the anterior descending left coronary artery. At the midpoint, ligation was conducted using a 6-0 suture. The operators directly observed that the anterior and apical left ventricular myocardium gradually turned to a dull and even pale color, which was accompanied with a weakened pulse. ECG showed a gradual ST segment elevation in I and $\mathrm{aVL}$, indicating the formation of MI. Fully intraoperative hemostasis was achieved, followed by a layer-by-layer sternal closure subsequent to the ligation. For the $\mathrm{S}$ group, a suture was placed under the left anterior descending coronary artery without ligation. To prevent postoperative wound infection, all the rabbits were injected with penicillin at $100,000 \mathrm{U} / \mathrm{d}$ for 3 consecutive days.

Detection of the left ventricular ejection fraction (LVEF) and left atrial diameter ( $L A D)$. Cardiac ultrasound was performed 8 weeks postoperatively using an ultrasound probe $(5-12 \mathrm{MHz})$. The left ventricular end-systolic diameter, end-diastolic diameter, LVEF and LAD were measured. Real-time video recorded during the measuring was documented for future analysis. The final data represented the average of 3 parallel measurements.

Specimen collection. Subsequent to measuring their weights 8 weeks postoperatively, the rabbits were anesthetized by intraperitoneal injection of $3 \%$ sodium pentobarbital (40 mg/kg), followed by thoracotomy. The heart was quickly harvested and rinsed with saline. The large blood vessels, right atrium, left ventricle and right ventricle were removed. The left atrium was separated and divided into two portions; one portion was stored at $-80^{\circ} \mathrm{C}$ for the measurement of collagen and type I collagen protein levels and another portion was fixed with $4 \%$ paraformaldehyde, embedded in paraffin and sectioned for hematoxylin and eosin (HE) and Masson staining.

Measurement of muscle hydroxyproline and collagen content. The content of myocardial hydroxyproline was measured to derive the content of myocardial collagen. First, 30-50 mg of left atrial tissue was homogenized and processed according to the manufacturer's instructions provided with the hydroxyproline assay kit. Subsequently, the supernatant in each tube was collected and the optical density (OD) value at $550 \mathrm{~nm}$ was measured with a spectrophotometer. The content of collagen (mg/l) was calculated according to the following formula: Collagen content $=[(\mathrm{OD}$ value of the sample tube $-\mathrm{OD}$ value of the blank tube) / (OD value of the standard tube - OD value of the blank tube)] x $7.46 \times$ dilution factor.

Detection of type I collagen expression in left atrial myocardial tissue by western blot analysis. The tissue stored at $-80^{\circ} \mathrm{C}$, as mentioned in 'Specimen collection,' was weighed and dissolved in lysate buffer. The proteins were extracted, quantified, separated by SDS-PAGE electrophoresis and electrotransferred following denaturation. The nitrocellulose (NC) membrane bearing the transferred proteins was incubated with the blocking solution at room temperature for $1 \mathrm{~h}$. Subsequently, the NC membrane was placed in a hybridization bag, agitated in the primary antibody solution at room temperature for $30 \mathrm{~min}$ and incubated in a $4^{\circ} \mathrm{C}$ refrigerator overnight. Subsequent to washing the polyvinylidene fluoride (PVDF) membrane with Tris-Buffered saline and Tween-20 (TBST) 3 times, with $10 \mathrm{~min}$ for each wash, the secondary antibody was added, followed by an incubation at room temperature for $60 \mathrm{~min}$. Subsequent to washing with TBST 3 times for $10 \mathrm{~min}$ each, the PVDF membrane was developed using chromogenic substrate solution in a darkroom and the image was captured to measure the gray value for data analysis. The GAPDH level served as the internal control.

Statistical analysis. The SPSS version 17.0 (SPSS, Inc., Chicago, IL, USA) statistical package was applied for statistical analysis. All the data are expressed as mean \pm standard deviation. Comparisons among multiple groups were performed using an analysis of variance and pairwise comparisons were carried out using the least significant difference method. $\mathrm{P}<0.05$ was considered to indicate a statistically significant difference.

\section{Results}

Atrial myocytes histomorphological changes of the rabbits in each group. HE staining showed that the atrial myocytes in the $\mathrm{S}$ group were arranged regularly and that infiltration of a small amount of inflammatory cells had occurred, although neither myocardial necrosis and hypertrophy nor stromal hyperplasia was observed. In the MI group, the number of atrial myocytes was decreased, their arrangement was irregular, the nuclei were of different sizes, with a few dissolved or ruptured, and there was an accumulation of interstitial collagen. Compared to the $\mathrm{MI}$ group, the $\mathrm{R}_{\mathrm{s}}$ and $\mathrm{R}_{\mathrm{l}}$ groups exhibited reduced myocardial necrosis and reduced infiltration of inflammatory cells, with a significant improvement in compensatory hypertrophy, stromal hyperplasia and collagen accumulation in myocardial cells, as shown in Fig. 1. Masson staining showed normal cardiac tissue stained in red and collagen fiber stained in blue. The expression of collagen fiber in the left atrial tissue of MI rabbits significantly increased, with fibrosis and interstitial collagen accumulation in certain cardiomyocytes, as shown in Fig. 2.

Comparison of LAD, LVEF and cardiomyocyte collagen content in the rabbits. The LAD and the left atrial collagen content in the MI group were significantly greater than those observed in the $\mathrm{S}$ group $(\mathrm{P}<0.01)$, while the $\mathrm{LVEF}$ value in the MI group was significantly lower than that in the $\mathrm{S}$ group $(\mathrm{P}<0.01)$. The LAD and the left atrial collagen content in the $\mathrm{R}_{\mathrm{s}}$ and $\mathrm{R}_{1}$ groups were significantly lower than those in the MI group $(\mathrm{P}<0.01)$, while the LVEF values in these groups were significantly higher than that in the MI group $(\mathrm{P}<0.01)$. Compared to the $\mathrm{R}_{\mathrm{s}}$ group, all the indicators in the $\mathrm{R}_{1}$ group were significantly lower, although the differences were not statistically significant, as shown in Table I.

Expression of left atrial type I collagen. The expression of left atrial type I collagen in the MI group was significantly higher than that observed in the $\mathrm{S}$ group $(\mathrm{P}<0.01)$ and the expression of left atrial type I collagen in the $R_{s}$ and $R_{1}$ groups 

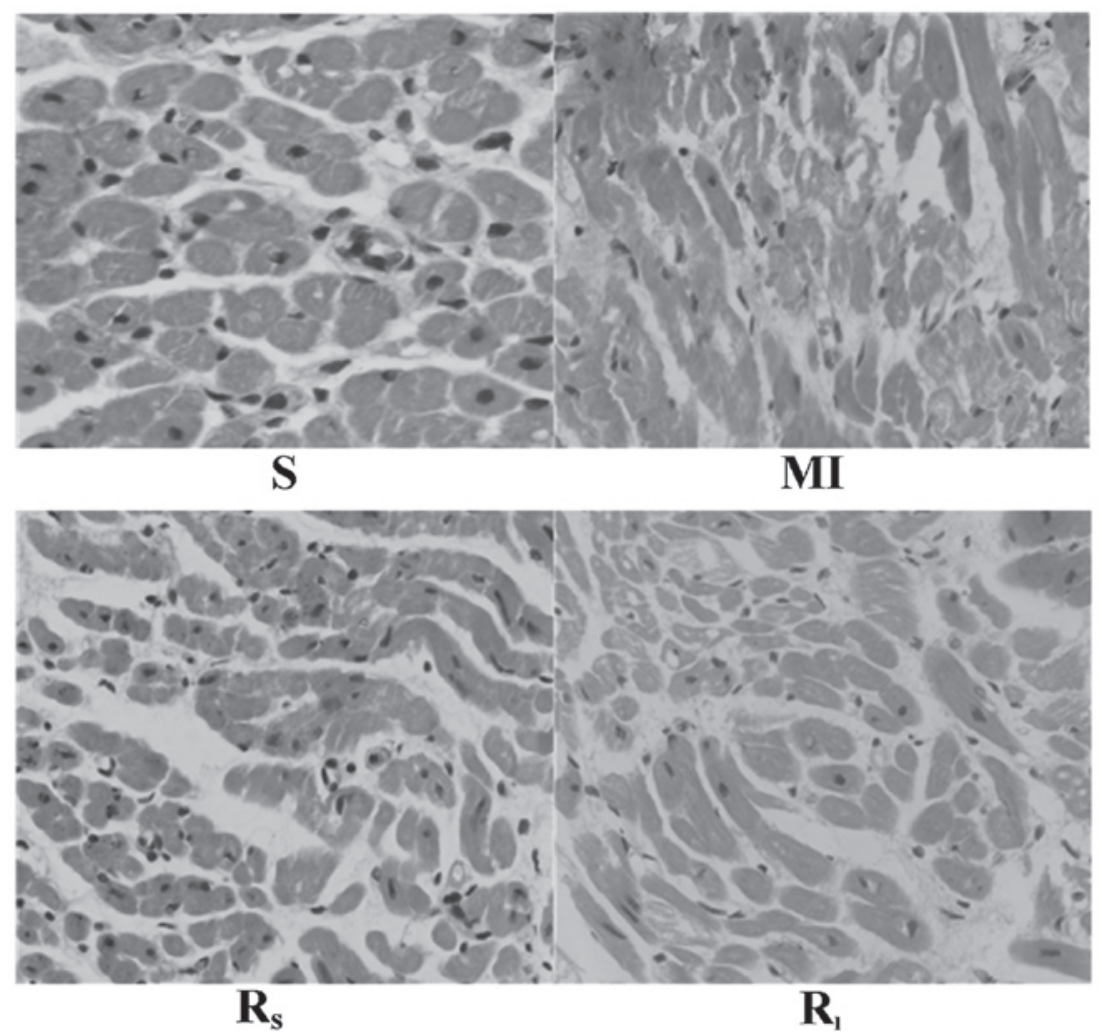

Figure 1. Hematoxylin and eosin staining of left atrial tissues in the sham operation (S), myocardial infarction (MI), low-dose rosuvastatin ( $\left.\mathrm{R}_{\mathrm{s}}\right)$ and high-dose rosuvastatin $\left(\mathrm{R}_{1}\right)$ groups (magnification, $\mathrm{x} 400$ ).
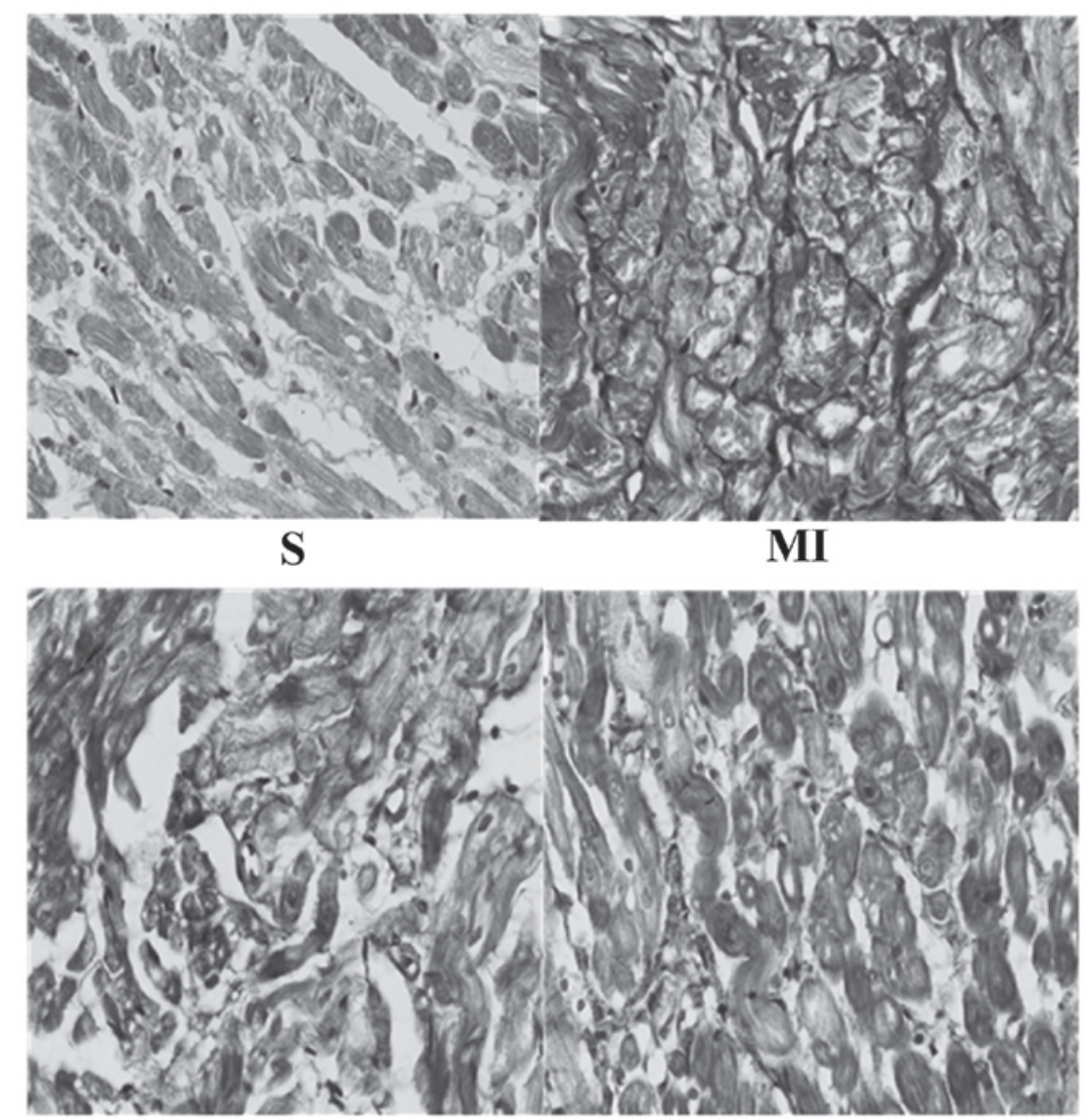

$\mathbf{R}_{\mathbf{s}}$

$\mathbf{R}_{\mathbf{1}}$

Figure 2. Masson staining of left atrial tissues in the sham operation (S), myocardial infarction (MI), low-dose rosuvastatin $\left(\mathrm{R}_{\mathrm{s}}\right)$ and high-dose rosuvastatin $\left(\mathrm{R}_{\mathrm{l}}\right)$ groups (magnification, $\mathrm{x} 400$ ). 
Table I. Effect of rosuvastatin on left atrial diameter (LAD), left ventricular ejection fraction (LVEF) and collagen content in the rabbits with myocardial infarction (MI).

\begin{tabular}{llccc}
\hline Indexes & S group & MI group & $\mathrm{R}_{\mathrm{s}}$ group & $\mathrm{R}_{1}$ group \\
\hline Quality of the heart, g & $4.01 \pm 0.56$ & $8.12 \pm 1.11$ & $0.71 \pm 0.10$ & $21.32 \pm 3.46$ \\
LAD, mm & $6.98 \pm 0.62^{\mathrm{a}}$ & $11.22 \pm 1.51^{\mathrm{a}}$ & $0.36 \pm 0.11^{\mathrm{a}}$ & $29.79 \pm 5.50^{\mathrm{a}}$ \\
LVEF, \% & $5.01 \pm 0.37^{\mathrm{b}}$ & $9.85 \pm 1.60^{\mathrm{b}}$ & $0.64 \pm 0.10^{\mathrm{b}}$ & $25.15 \pm 3.59^{\mathrm{b}}$ \\
Collagen, mg/l & $4.86 \pm 0.41^{\mathrm{b}}$ & $9.28 \pm 0.99^{\mathrm{b}}$ & $0.62 \pm 0.09^{\mathrm{b}}$ & $24.59 \pm 3.58^{\mathrm{b}}$ \\
\hline
\end{tabular}

${ }^{a} \mathrm{P}<0.05$, MI group vs. $\mathrm{S}$ group; ${ }^{b} \mathrm{P}<0.05, \mathrm{R}_{\mathrm{s}}$ and $\mathrm{R}_{1}$ groups vs. MI group. Data are presented as mean \pm standard deviation. $\mathrm{S}$, sham operation; $\mathrm{R}_{\mathrm{s}}$, low-dose rosuvastatin; $\mathrm{R}_{1}$, high-dose rosuvastatin.

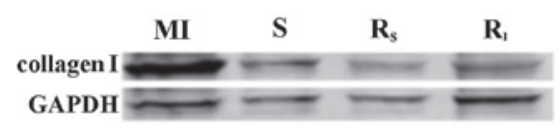

Figure 3. Expression of left atrial type I collagen in the myocardial infarction (MI), low-dose rosuvastatin $\left(\mathrm{R}_{\mathrm{s}}\right)$, high-dose rosuvastatin $\left(\mathrm{R}_{1}\right)$ and sham operation (S) groups. Western blot analysis of left atrial type I collagen of the groups. GAPDH was used as a control. Collagen I, left atrial type I collagen.

was significantly lower compared to the MI group $(\mathrm{P}<0.05)$. However, the difference between the $\mathrm{R}_{\mathrm{s}}$ and $\mathrm{R}_{1}$ groups was not statistically significant $(\mathrm{P}>0.05)$, as shown in Fig. 3.

\section{Discussion}

Atrial structural remodeling involves changes in cells and the extracellular matrix and is most prominently manifested by atrial interstitial fibrosis (6). Interstitial fibrosis mainly occurs in the extracellular matrix and collagen is the most important component of the myocardial extracellular matrix, which is dominated by type I collagen in the heart. The present study revealed the following results: i) The atrial myocytes of rabbits in the MI group showed hypertrophy and hyperplasia, irregular arrangement, karorrhexis and karyolysis, as well as deformation and necrosis; ii) the MI groups also revealed accumulations of interstitial collagen and significantly increased biomarkers of myocardial fibrosis, such as myocardial collagen and the expression of type I collagen; and iii) significant expansion of the LAD was observed in the MI group. These results indicated that atrial structural remodeling occurred following MI and this remodeling may be associated with the following aspects. First, ventricular systolic function is severely impaired subsequent to MI, as supported by the data in the present study showing that the LVEF value of the MI rabbits was significantly reduced. This impairment in ventricular systolic function may further lead to ventricular diastolic dysfunction, thereby causing a high left atrial pressure and atrial hypertrophy and ultimately inducing atrial remodeling. Second, the levels of myocardial matrix metalloproteinase (MMP)-3 and -9 and tissue inhibitor of metalloproteinase are upregulated following MI (9). This increase in MMP expression is associated with the metabolic dysregulation of extracellular myocardial interstitial type I collagen and the degradation of collagen, which can promote atrial fibrosis and lead to the occurrence of post-MI atrial remodeling. Third, excessive oxidative stress is an important driving factor of myocardial remodeling (10) and the oxidative stress of cardiomyocytes increases following MI, thereby promoting atrial structural remodeling. Atrial structural remodeling can not only impair atrial systolic and diastolic function but also provide a matrix for impulse reentry, leading to AF and other atrial arrhythmias. In addition, a number of studies have also shown that the excessively high pressure and sharp dilatation of the left atrium following MI increases the susceptibility to $\operatorname{AF}(11,12)$.

A previous study has also confirmed that statins have anti-arrhythmia effects and can improve post-MI cardiac remodeling (13). In this study, after applying rosuvastatin treatment for 8 weeks, the structure and morphology of impaired atrial cells were significantly improved, indicating that rosuvastatin effectively improved atrial structural remodeling following MI. The possible mechanisms for the beneficial effects of statins on atrial structural remodeling after MI include the following: i) Statins can activate the peroxisome proliferator-activated receptors- $\alpha$ and $-\gamma$, extracellular MMP-9 and tissue protein S, thereby inhibiting cardiac hypertrophy and the development of fibrosis (14); ii) statins can reduce the type I collagen level in cardiomyocytes and fibrotic regions by inhibiting transforming growth factor- $\beta$, which is an important factor promoting myocardial fibrosis, affecting the production of extracellular matrix by inducing the expression of type I collagen and fibronectin (15); and iii) the results of the present study showed that the LVEF value increased after rosuvastatin treatment in MI rabbits, indicating that rosuvastatin plays a role in improving impaired left ventricular systolic function. The study by Tsai et al (16) also showed that statins inhibited the renin-angiotensin-aldosterone system to reduce ventricular remodeling and improve impaired left ventricular systolic function following MI, thereby reducing high left atrial pressure and left atrial hypertrophy. Taken together, these results indicate that rosuvastatin can not only directly improve atrial structural remodeling but also indirectly improve atrial structural remodeling by reversing ventricular structural remodeling following MI. In addition, statins can mitigate atrial remodeling by inhibiting oxidative stress pathways, such as those mediated by guanylate protein A/guanylate kinase (17). Thus, statin-mediated improvement of atrial structural remodeling following MI may represent an important mechanism underlying their preventive and therapeutic effects on atrial arrhythmia following MI, particularly for AF. 
The present study also investigated the effects of rosuvastatin on atrial remodeling at different doses. Although the results of corresponding indicators revealed that a high dose of rosuvastatin could partly improve atrial structural remodeling than a low dose, the differences were not statistically significant. This finding may be associated with the short treatment time or the insufficient dose difference between groups.

\section{Acknowledgements}

The authors thank the American Journal Experts (AJE) for their helpful critical reading of this manuscript and have received an AJE 'Editorial Certificate' for language editing. The present study was supported by the National Natural Science Foundation of China (grant no. 81270237) and the Foundation of Shandong Province (grant no. 2012ZRB14226 and no. ZR2010HL008).

\section{References}

1. Abuissa H,O'Keefe JH and Bybee KA: Statins as anti-arrhythmics: a systematic review part II: effects on risk of ventricular arrhythmias. Clin Cardiol 32: 549-552, 2009.

2. Ma YX, Li WH and Xie Q: Rosuvastatin inhibits TGF-beta1 expression and alleviates myocardial fibrosis in diabetic rats Pharmazie 68: 355-358, 2013.

3. Paraskevaidis IA, Iliodromitis EK, Ikonomidis I, et al: The effect of acute administration of statins on coronary microcirculation during the pre-revascularization period in patients with myocardial infraction. Atherosclerosis 223: 184-189, 2012.

4. Hayashidani S, Tsutsui H, Shiomi T, et al: Fluvastatin, a 3-hydroxy-3-methylglutaryl coenzyme a reductase inhibitor, attenuates left ventricular remodeling and failure after experimental myocardial infarction. Circulation 105: 868-873, 2002.

5. Jiang BH, Tardif JC, Sauvageau S, et al: Beneficial effects of atorvastatin on lung structural remodeling and function in ischemic heart failure. J Card Fail 16: 679-688, 2010

6. Allessie M, Ausma J and Schotten U: Electrical, contractile and structural remodeling during atrial fibrillation. Cardiovasc Res 54: 230-246, 2002.
7. Pinho-Gomes AC, Reilly S, Brandes RP and Casadei B: Targeting inflammation and oxidative stress in atrial fibrillation: role of 3-hydroxy-3-methylglutaryl-coenzyme a reductase inhibition with statins. Antioxid Redox Signal 20: 1268-1285, 2014.

8. Kumagai K, Nakashima H and Saku K: The HMG-CoA reductase inhibitor atorvastatin prevents atrial fibrillation by inhibiting inflammation in a canine sterile pericarditis model. Cardiovasc Res 62: 105-111, 2004.

9. Aronson D, Mutlak D, Bahouth F, et al: Restrictive left ventricular filling pattern and risk of new-onset atrial fibrillation after acute myocardial infarction. Am J Cardiol 107: 1738-1743, 2011.

10. McCarty MF: Practical prevention of cardiac remodeling and atrial fibrillation with full-spectrum antioxidant therapy and ancillary strategies. Med Hypotheses 75: 141-147, 2010.

11. Alasady M, Shipp NJ, Brooks AG, et al: Myocardial infarction and atrial fibrillation: importance of atrial ischemia. Circ Arrhythm Electrophysiol 6: 738-745, 2013.

12. Bode F, Katchman A, Woosley RL and Franz MR: Gadolinium decreases stretch-induced vulnerability to atrial fibrillation. Circulation 101: 2200-2205, 2000.

13. Zhang J, Xu Y, Pan L, Chen T, Chen Z and Zhao R: Effect of simvastatin on collagen I deposition in non-infarcted myocardium: role of $\mathrm{NF}_{-} \kappa \mathrm{B}$ and osteopontin. Can J Physiol Pharmacol 88: 1026-1034, 2010

14. Qin YM, Ye P, He JQ, Sheng L, Wang LY and Du J: Simvastatin inhibited cardiac hypertrophy and fibrosis in apolipoprotein E-deficient mice fed a 'Western-style diet' by increasing PPAR $\alpha$ and $\gamma$ expression and reducing TC, MMP-9, and Cat S levels. Acta Pharmacol Sin 31: 1350-1358, 2010.

15. Shyu KG, Wang BW, Chen WJ, Kuan P and Hung CR: Mechanism of the inhibitory effect of atorvastatin on endoglin expression induced by transforming growth factor-betal in cultured cardiac fibroblasts. Eur J Heart Fail 12: 219-226, 2010.

16. Tsai CT, Lai LP, Kuo KT, et al: Angiotensin II activates signal transducer and activators of transcription 3 via Rac1 in atrial myocytes and fibroblasts: implication for the therapeutic effect of statin in atrial structural remodeling. Circulation 117: 344-355, 2008.

17. Briones AM, Rodriguez-Criado N, Hernanz R, et al: Atorvastatin prevents angiotensin II-induced vascular remodeling and oxidative stress. Hypertension 54: 142-149, 2009. 